Original article

\title{
Assessment of the occupational stress among nuclear medicine technical professionals in India
}

\author{
Sanny Bhushan Kumar ${ }^{a}$, Kevin Fonseca ${ }^{a}$, Ashwani Verma ${ }^{\text {b,c, }}$, Ravi Ranjan Kumar ${ }^{\mathrm{d}}$

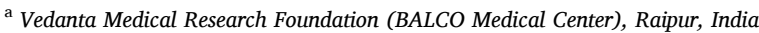 \\ ${ }^{\mathrm{b}}$ Ministry of Health and Family Welfare, Nirman Bhawan, New Delhi, India \\ ${ }^{\mathrm{c}}$ DIT University, Uttarakhand, India \\ ${ }^{\mathrm{d}}$ Centre for Nuclear Medicine, Panjab University, Chandigarh, India
}

\section{A R T I C L E I N F O}

\section{Keywords:}

Stress

Occupational stress

Nuclear medicine

Healthcare professionals

\begin{abstract}
A B S T R A C T
Background: Occupational stress has become subject of focus for studies involving healthcare professionals and it is a serious health issue. Occupational stress can lead to several negative consequences not only to the professionals but also for those who directly or indirectly deal or involve with them. This study was conducted to assess the occupational stress among nuclear medicine technical professionals across the country. Materials and methods: The study was conducted in 2 phases: Phase I involved the modification and validation of the questionnaire, whereas phase II involved data collection. A cross-sectional, time-bound web-based study using convenience sampling was carried out in India. Data was collected using Nuclear Medicine Occupational Stress Questionnaire (NMOSQ) via google forms. Data was analysed using SPSS 16. Data was normally distributed and mean and standard deviations were reported for continuous data. Chi square test was used to find association between categorical variables.

Results: Out of 450, 225 nuclear medicine technical professionals responded, yielded a response rate of 51\%. Prevalence of stress among nuclear medicine technical profession was 89\%; higher in Indian setting. Different domains of NMOSQ exhibited different levels of stress among study population.

Conclusion: This study helps to identify certain factors that contribute towards work-related stress. These in turn have an impact on the overall professional output of a professional.
\end{abstract}

\section{Introduction}

Occupational Stress may be defined as a situation which forces a person to deviate from its routine functioning due to change in psychological or physiological condition. ${ }^{1}$ According to World Health Organization, work-related or occupational stress is the response when individuals are presented with work demands and pressures that are unmatched to their knowledge and abilities and which challenge their ability to cope. ${ }^{2}$ Stress occurs in a wide range of work circumstances but is often made worse when individuals have little support from peers at work. Pressure at the workplace is inevitable due to the demands of the work environment and responsibilities. However, when pressure at work becomes excessive or otherwise unmanageable, it leads to occupational stress. ${ }^{2}$ It can ultimately affect an individual's physical and psychological health which ultimately affects the business performance. $^{3}$
Work-related stress could be due to poor work organization and work allocation, or lack of control over work processes, unsatisfactory working conditions and lack of support from colleagues and supervisors. ${ }^{2}$ The type of job, role in the organization, the personality of the professionals, the interpersonal relationships within organization and work-life balance are potential sources of stress among health care professionals. ${ }^{4}$ Several studies have shown high level of stress among healthcare professionals because of their work which directly hampers their ability to provide high quality care to patients. ${ }^{5-9,20}$ In Covid-19 pandemic, recent evidence suggested that there is an increased risk of acquiring stress related disorder among healthcare workers. ${ }^{10,11}$

Nuclear medicine is a branch of medicine that uses radioactive tracers (radiopharmaceuticals) to assess bodily functions and to diagnose and treat diseases. Nuclear Medicine technical professionals have to deal with certain responsibilities such as procurement of radioactive medicine, preparation of radiopharmaceuticals, quality

\footnotetext{
* Corresponding author. DIT University, Uttarakhand, India

E-mail address: vamit1989@gmail.com (A. Verma).
} 
control of equipment's, patient scheduling, patient scanning, radiation safety etc. ${ }^{12}$ Previous evidence from the United States suggested that occupational stress experienced by Nuclear medicine allied health discipline were due to equipment malfunctions, add-on examinations, uncooperative physicians, lack of staff, and uncooperative patients. ${ }^{13,14}$ In developed countries, there is a greater awareness and sensitivity to the problems that are faced by healthcare professionals whereas there is a healthcare workers are not immune to psychological consequences in developing countries. ${ }^{15}$ But there is a paucity of literature from developing countries like India on occupational stress among nuclear medicine technical professionals and associated risk factors for stress. Hence, this study was conducted with an objective to assess the prevalence of occupational stress and to identify the specific occupational stressors among nuclear medicine technical professionals across the country.

\section{Methods}

This study was conducted in accordance with the Helsinki declaration. ${ }^{16}$ All participants were explained about the purpose of study and their participation was voluntary. Confidentiality of the study participants was maintained throughout the study by making the participants' information anonymous. An informed consent was obtained from each participant prior to their participation in this study. The present study was conducted in two phases; Phase I involved the modification and validation of the questionnaire, whereas phase II involved online data collection.

\subsection{Phase I: modification and validation of the questionnaire}

A pre-tested and validated Nuclear Medicine Occupational Stress Questionnaire (NMOSQ) ${ }^{14}$ was modified through expert discussion and their opinion for Indian context. The modified questionnaire was then given to five experts in the field of nuclear medicine with more than five years of experience in clinical setting and research for content validation. All experts rated each question of the questionnaire using a rating scale of irrelevant, somewhat relevant, quite relevant, and relevant. The items rated either relevant or quite relevant were incorporated in the final questionnaire. A score of 0.9 was obtained on the content validity index for Scale (S-CVI) for the final questionnaire, which indicated the excellent content validity. ${ }^{17}$ All questions proposed in modified version of the questionnaire were retained in the final version.

The questionnaire used in this study was consisted of two sections: demographics and content questions. Demographic variables included age, gender, designation, qualification, institution type, year of work experience, workplace set up and number of qualified technical nuclear professional. The second section of the questionnaire consisted of content questions based on 24 domains.

The domains are malfunctioning of equipment, lack of radiation safety equipment, non-cooperative patients, handling paediatric patients, death of the patients, preparation of patients, scheduling patients, work load, working hours, documentation work, occupational related health illness, random regulatory inspections, stress faced during infrequently performed studies, radiation dose received from occupational radiation exposure, learning new protocols \& procedures, lack of supporting staff cooperation, lack of cooperation with other nuclear medicine technical professionals, lack of cooperation with nuclear medicine physician, lack of nuclear medicine technical professionals, institute management pressure, incomplete procedure requisition form, salary issues and uncertainty of job security. These domains were rated on a five-point Likert scale, which ranges from "No stress" to "Extremely severe stress". No stress was coded as " 0 ", mild stress was coded as " 1 ", moderate stress was coded as " 2 ", severe stress was coded as " 3 " and extremely severe stress was coded as " 4 ". For the data analysis and overall scoring, "No stress" was considered as "Stress Absent" and from "mild stress to extremely severe stress" considered as "Stress Present".

\subsection{Phase II: Data collection and analysis}

An online, cross sectional study design was used to collect data from nuclear medicine technical professionals practicing in India and possessing a minimum qualification of bachelor's degree using convenience sampling.

\subsection{Inclusion criteria}

Nuclear medicine professionals who are registered with Nuclear medicine physicist association and Society of Nuclear Medicine, India and practicing in India are eligible to participate in this survey.

\subsection{Exclusion criteria}

Nuclear medicine professionals who do not consent to participate in this survey.

\subsection{Data collection}

The finalized questionnaire was then made available using google form along with email link. This questionnaire link was sent via email to 450 nuclear medicine technical professionals working in India, randomly chosen from Nuclear medicine physicist association and Society of Nuclear Medicine, India. The responses were recorded into Google forms, the identity of the respondents was kept anonymous, and these responses were made accessible to the principal investigator only. No personal information was collected to maintain anonymity.

The data collection was carried out from 9th to 18th August, 2020. The google form includes the brief description of the study and formal consent. Individuals who consented to participate were proceeded for next process. The questionnaire was administered in English language and took approximately $7 \mathrm{~min}$ for its completion. Data was entered into Microsoft Excel and cross checked for presence of any inaccuracy to maintain its accuracy and validity. Only principal investigator has the access to this raw data to maintain confidentiality.

\subsection{Data analysis}

Descriptive statistics comprised of mean and standard deviations were reported for continuous variables and frequency and percentage for discrete variables. The responses obtained on the NMOSQ were summarized with cross-tabulation using SPSS 16 (SPSS Inc., Chicago IL). Chi square test was used to find association between categorical variables. A p-value of less than 0.05 was considered as statistically significant.

\section{Result}

Out of 450 nuclear medicine technical professionals who were contacted, 231 had responded. Of these 231 participants, 6 participants had declined to provide consent, and 225 participants have provided their voluntary consent for participation and completed the survey with the participation rate of $51.3 \%$.

\subsection{Participant characteristics}

The mean age of participants was $30.2 \pm 7.4$ years, ranges $21-59$ years. Majority of participants (62.7\%) were in between 21 and 30 years of age, nearly one-fourth $(26.7 \%)$ belongs to age group of 31-40 years whereas only $7.6 \%$ and $3.1 \%$ participants were in between 41 and 50 years and above 51 years respectively. Male participants (57\%) were slightly more than the female participants (43\%). Educational profile depicted that more than three-fourth of the participants (79.1\%) were postgraduate in nuclear medicine followed by $17.8 \%$ participants who were only graduates and $3.1 \%$ were holding $\mathrm{PhD}$. In terms of 
occupation, more than half $(51.1 \%)$ were Nuclear Medicine Technologist, followed by Radiation safety officer $(22.2 \%)$. More than half (56.2\%) participants felt to be stressed when they are engaged with private hospitals as compared to government hospitals (25.9\%) and diagnostic centre (17.9\%). Nearly three-fourth $(73.1 \%)$ participants who are involved with multi-modality setup are stressed as compared to the participants working in single modality setup (26.9\%). Nearly $62 \%$ participant reported as stressed when there is only single qualified technical nuclear medicine professional than presence of multiple qualified technical nuclear medicine professional (37.8\%).

Table 1 summarizes the sociodemographic characteristics of study participants.

Table 1

Socio demographic characteristics of study participants.

\begin{tabular}{|c|c|c|c|c|c|}
\hline \multicolumn{2}{|c|}{ Socio demographic variables } & \multicolumn{2}{|c|}{ Overall stress } & \multirow[t]{2}{*}{ Total } & \multirow{2}{*}{$\begin{array}{l}\mathrm{P} \\
\text { value }\end{array}$} \\
\hline & & $\begin{array}{l}\text { Yes, n } \\
(\%)\end{array}$ & $\begin{array}{l}\text { No, n } \\
(\%)\end{array}$ & & \\
\hline \multirow[t]{4}{*}{$\begin{array}{l}\text { Age groups (in } \\
\text { years) }\end{array}$} & $21-30$ & $\begin{array}{l}127 \\
(63.2)\end{array}$ & $\begin{array}{l}14 \\
(58.3)\end{array}$ & $\begin{array}{l}141 \\
(62.7)\end{array}$ & 0.312 \\
\hline & $31-40$ & $\begin{array}{l}51 \\
(25.4)\end{array}$ & $\begin{array}{l}9 \\
(37.5)\end{array}$ & $\begin{array}{l}60 \\
(26.7)\end{array}$ & \\
\hline & $41-50$ & $16^{8}$ & $1(4.2)$ & $\begin{array}{l}17 \\
(7.6)\end{array}$ & \\
\hline & $51-60$ & $7(3.5)$ & $0(0)$ & $7(3.1)$ & \\
\hline \multirow[t]{3}{*}{ Qualification } & Graduate & $\begin{array}{l}33 \\
(16.4)\end{array}$ & $\begin{array}{l}7 \\
(29.2)\end{array}$ & $\begin{array}{l}40 \\
(17.8)\end{array}$ & 0.072 \\
\hline & Postgraduate & $\begin{array}{l}163 \\
(81.1)\end{array}$ & $\begin{array}{l}15 \\
(62.5)\end{array}$ & $\begin{array}{l}178 \\
(79.1)\end{array}$ & \\
\hline & Phd & $5(2.5)$ & $2^{8}$ & $7(3.1)$ & \\
\hline \multirow[t]{10}{*}{ Designation } & $\begin{array}{l}\text { Associate } \\
\text { Professor }\end{array}$ & $2^{1}$ & $0(0)$ & $2(0.9)$ & 0.838 \\
\hline & $\begin{array}{l}\text { Assistant } \\
\text { Professor }\end{array}$ & $2^{1}$ & $0(0)$ & $2(0.9)$ & \\
\hline & Scientific Officer & $6^{3}$ & $0(0)$ & $6(2.7)$ & \\
\hline & RSO & $\begin{array}{l}45 \\
(22.4)\end{array}$ & $\begin{array}{l}5 \\
(20.8)\end{array}$ & $\begin{array}{l}50 \\
(22.2)\end{array}$ & \\
\hline & $\begin{array}{l}\text { Nuclear Medicine } \\
\text { Physicist }\end{array}$ & $\begin{array}{l}26 \\
(12.9)\end{array}$ & $6(25)$ & $\begin{array}{l}32 \\
(14.2)\end{array}$ & \\
\hline & Medical Physicist & $\begin{array}{l}11 \\
(5.5)\end{array}$ & $1(4.2)$ & $\begin{array}{l}12 \\
(5.3)\end{array}$ & \\
\hline & Radiochemist & $1(0.5)$ & $0(0)$ & $1(0.4)$ & \\
\hline & Nuclear Medicine & 103 & 12 & 115 & \\
\hline & Technologist & $(51.2)$ & $(50)$ & $(51.1)$ & \\
\hline & $\begin{array}{l}\text { Scientific } \\
\text { Assistant }\end{array}$ & $5(2.5)$ & $0(0)$ & $5(2.2)$ & \\
\hline \multirow[t]{3}{*}{ Institute Type } & Govt. Hospital & $\begin{array}{l}52 \\
(25.9)\end{array}$ & $6(25)$ & $\begin{array}{l}58 \\
(25.8)\end{array}$ & 0.196 \\
\hline & Private Hospital & $\begin{array}{l}113 \\
(56.2)\end{array}$ & $\begin{array}{l}17 \\
(70.8)\end{array}$ & $\begin{array}{l}130 \\
(57.8)\end{array}$ & \\
\hline & Diagnostic Centre & $\begin{array}{l}36 \\
(17.9)\end{array}$ & $1(4.2)$ & $\begin{array}{l}37 \\
(16.4)\end{array}$ & \\
\hline \multirow[t]{4}{*}{$\begin{array}{c}\text { Year of Work } \\
\text { Experience }\end{array}$} & Less than 5 years & $\begin{array}{l}119 \\
(59.2)\end{array}$ & $\begin{array}{l}15 \\
(62.5)\end{array}$ & $\begin{array}{l}134 \\
(59.6)\end{array}$ & 0.704 \\
\hline & $5-10$ years & $\begin{array}{l}40 \\
(19.9)\end{array}$ & $6(25)$ & $\begin{array}{l}46 \\
(20.4)\end{array}$ & \\
\hline & 10-15 years & $\begin{array}{l}23 \\
(11.4)\end{array}$ & $1(4.2)$ & $\begin{array}{l}24 \\
(10.7)\end{array}$ & \\
\hline & $\begin{array}{l}\text { More than } 15 \\
\text { years }\end{array}$ & $\begin{array}{l}19 \\
(9.5)\end{array}$ & $2(8.3)$ & $\begin{array}{l}21 \\
(9.3)\end{array}$ & \\
\hline Workplace set up & $\begin{array}{l}\text { Single Modality } \\
\text { Setup } \\
\text { Multi-Modality } \\
\text { Setup }\end{array}$ & $\begin{array}{l}54 \\
(26.9) \\
147 \\
(73.1)\end{array}$ & $\begin{array}{l}7 \\
(29.2) \\
17 \\
(70.8)\end{array}$ & $\begin{array}{l}61 \\
(27.1) \\
164 \\
(72.9)\end{array}$ & 0.811 \\
\hline $\begin{array}{l}\text { Number of } \\
\text { Qualified }\end{array}$ & One & $\begin{array}{l}124 \\
(61.7)\end{array}$ & $\begin{array}{l}16 \\
(66.7)\end{array}$ & $\begin{array}{l}140 \\
(62.2)\end{array}$ & 0.635 \\
\hline $\begin{array}{l}\text { Technical } \\
\text { Nuclear } \\
\text { medicine } \\
\text { Professional }\end{array}$ & More than one & $\begin{array}{l}77 \\
(37.8)\end{array}$ & $\begin{array}{l}8 \\
(33.3)\end{array}$ & $\begin{array}{l}85 \\
(37.8)\end{array}$ & \\
\hline
\end{tabular}

\subsection{Prevalence of occupational stress among study participants}

Overall, prevalence of stress among nuclear medicine technical professionals is $89.3 \%$. It is also seen that males (89.9\%) have slightly higher occupational stress than females $(88.5 \%)$ but this difference is is not statistically significant ( $\mathrm{p}$ value $=0.452$ ).

Table 2 reports the overall mean score of different domains based on NMOSQ. Higher the mean of a domain depicts the higher stress among study participants. The five domains where study participants experienced most stress were death of patients, lack of supporting staff cooperation, institute management pressure, lack of radiation safety equipment, uncertainty of job security. The five domains where participants reported least stress were learning new protocols \& procedures, preparation of patients, scheduling patients, random regulatory inspection and documentation work.

Both gender experienced stress in majority of domains except for preparation of patients and in learning new protocols and procedures. Proportion of males experienced more stress is higher than females but there is no statistically significant difference between gender in any domain. Participants within the age group of 21-30 years (more than $50 \%$ ) experienced more stress as compared to other age categories. There is a statistically significant difference in between age groups for workload ( $\mathrm{p}$ value $<0.05$ ).

Table 3 reported the qualification wise stress among study participants for each domain. Of all participants, more than three-fourth of the participants with postgraduation degree experienced stress for each domain but is not statistically significant for majority of domains however there is a statistically significant difference of few domains like preparation of patients, documentation of work, learning new protocols and procedures with $\mathrm{p}$ value $<0.05$.

As observed in Table 4, stress is prevalent with the year of experience among study participants. More than half of the study participants having an experience of 10-15 years experienced less stress as compared to other participants for majority of domains. In a single domain of malfunction of equipment, participants with less than 5 years of experience feeling more stress as compared to other participants. However, there is no statistically significant difference in any of the domain.

Table 5 describes the workplace wise stress among study participants for each domain. As reported in Table 5, participants who are involved in multi-modality setup experienced more stress as compared to single

Table 2

Mean and SD of stress for each domain of NMOSQ.

\begin{tabular}{ll}
\hline Domain & $\begin{array}{l}\text { Mean } \\
\text { (SD) }\end{array}$ \\
\hline Malfunctioning of equipment & $1.37(0.9)$ \\
Non cooperative Patients & $1.46(0.9)$ \\
Workload & $1.44(1.0)$ \\
Handling Paediatric Patients & $1.31(1.0)$ \\
Death of the Patients & $1.77(1.2)$ \\
Preparation of Patients & $0.55(0.8)$ \\
Scheduling Patients & $0.80(0.9)$ \\
Working Hours & $1.01(1.0)$ \\
Radiation dose received from occupational radiation exposure & $1.27(1.0)$ \\
Random Regulatory Inspections & $0.98(0.9)$ \\
Stress faced during Infrequently performed studies & $1.06(0.9)$ \\
Learning new Protocols \& Procedures & $0.54(0.7)$ \\
Lack of Radiation Safety Equipment & $1.60(1.1)$ \\
Lack of Nuclear medicine Technical professionals & $1.53(1.1)$ \\
Lack of supporting staff cooperation & $1.65(1.1)$ \\
Lack of cooperation with Other Nuclear Medicine Technical & $1.33(1.1)$ \\
$\quad$ Professionals & \\
Lack of cooperation with Nuclear Medicine Physician & $1.55(1.1)$ \\
Institute Management Pressure & $1.63(1.0)$ \\
Documentation work & $0.99(0.9)$ \\
Incomplete Procedure Requisition Form & $1.24(0.9)$ \\
Salary issues & $1.45(1.1)$ \\
Occupational related Health illness & $1.18(1.0)$ \\
Uncertainty of Job security & $1.59(1.1)$ \\
\hline
\end{tabular}


Table 3

Qualification wise stress among study participants.

\begin{tabular}{|c|c|c|c|c|c|}
\hline \multirow[t]{2}{*}{ Domain } & \multirow[t]{2}{*}{ Stress } & \multicolumn{3}{|c|}{ Qualification } & \multirow{2}{*}{$\begin{array}{l}\mathrm{P} \\
\text { value }\end{array}$} \\
\hline & & Graduate & Postgraduate & $\mathrm{PhD}$ & \\
\hline \multirow[t]{2}{*}{$\begin{array}{l}\text { Malfunctioning of } \\
\text { equipment }\end{array}$} & Present & $31(16.8)$ & 147 (79.9) & $\begin{array}{l}6 \\
(3.3)\end{array}$ & 0.726 \\
\hline & Absent & $9(22)$ & $31(75.6)$ & $\begin{array}{l}1 \\
(2.4)\end{array}$ & \\
\hline \multirow[t]{2}{*}{$\begin{array}{l}\text { Lack of Radiation } \\
\text { Safety Equipment }\end{array}$} & Present & $34(19.7)$ & $134(77.5)$ & $\begin{array}{l}5 \\
(2.9)\end{array}$ & 0.395 \\
\hline & Absent & $6(11.5)$ & $44(84.6)$ & $\begin{array}{l}2 \\
(3.8)\end{array}$ & \\
\hline Non cooperative & Present & $35(17.8)$ & $156(79.2)$ & $6(3)$ & 0.989 \\
\hline Patients & Absent & $5(17.9)$ & $22(78.6)$ & $\begin{array}{l}1 \\
(3.6)\end{array}$ & \\
\hline \multirow[t]{2}{*}{$\begin{array}{l}\text { Handling Paediatric } \\
\text { Patients }\end{array}$} & Present & $30(16.9)$ & $142(80.2)$ & $\begin{array}{l}5 \\
(2.8)\end{array}$ & 0.716 \\
\hline & Absent & $10(20.8)$ & $36(75)$ & $\begin{array}{l}2 \\
(4.2)\end{array}$ & \\
\hline \multirow[t]{2}{*}{ Death of the Patients } & Present & $31(18.3)$ & $132(78.1)$ & $\begin{array}{l}6 \\
(3.6)\end{array}$ & 0.730 \\
\hline & Absent & $9(16.1)$ & $46(82.1)$ & $\begin{array}{l}1 \\
(1.8)\end{array}$ & \\
\hline \multirow[t]{2}{*}{ Preparation of Patients } & Present & $24(26.1)$ & $65(70.7)$ & $\begin{array}{l}3 \\
(3.3)\end{array}$ & $0.024^{\mathrm{a}}$ \\
\hline & Absent & $16(12)$ & $113(85)$ & $4(3)$ & \\
\hline \multirow[t]{2}{*}{ Scheduling Patients } & Present & $27(21.1)$ & $97(75.8)$ & $\begin{array}{l}4 \\
(3.1)\end{array}$ & 0.324 \\
\hline & Absent & $13(13.4)$ & $81(83.5)$ & $\begin{array}{l}3 \\
(3.1)\end{array}$ & \\
\hline \multirow[t]{2}{*}{ Work Load } & Present & $35(19.3)$ & $140(77.3)$ & $\begin{array}{l}6 \\
(3.3)\end{array}$ & 0.416 \\
\hline & Absent & $5(11.4)$ & $38(86.4)$ & $\begin{array}{l}1 \\
(2.3)\end{array}$ & \\
\hline \multirow[t]{2}{*}{ Working Hours } & Present & $25(18.9)$ & $103(78)$ & $4(3)$ & 0.862 \\
\hline & Absent & $15(16.1)$ & $75(80.6)$ & $\begin{array}{l}3 \\
(3.2)\end{array}$ & \\
\hline \multirow[t]{2}{*}{ Documentation work } & Present & $30(20.4)$ & $115(78.2)$ & $\begin{array}{l}2 \\
(1.4)\end{array}$ & $0.050^{\mathrm{a}}$ \\
\hline & Absent & $10(12.8)$ & $63(80.8)$ & $\begin{array}{l}5 \\
(6.4)\end{array}$ & \\
\hline \multirow{2}{*}{$\begin{array}{l}\text { Occupational related } \\
\text { Health illness }\end{array}$} & Present & $30(18.2)$ & $130(78.8)$ & $5(3)$ & 0.962 \\
\hline & Absent & $10(16.7)$ & $48(80)$ & $\begin{array}{l}2 \\
(3.3)\end{array}$ & \\
\hline \multirow[t]{2}{*}{$\begin{array}{l}\text { Random Regulatory } \\
\text { Inspections }\end{array}$} & Present & $30(20.4)$ & $113(76.9)$ & $\begin{array}{l}4 \\
(2.7)\end{array}$ & 0.345 \\
\hline & Absent & $10(12.8)$ & $65(83.3)$ & $\begin{array}{l}3 \\
(3.8)\end{array}$ & \\
\hline \multirow{2}{*}{$\begin{array}{l}\text { Stress faced during } \\
\text { Infrequently } \\
\text { performed studies }\end{array}$} & Present & $34(21)$ & $124(76.5)$ & $\begin{array}{l}4 \\
(2.5)\end{array}$ & 0.100 \\
\hline & Absent & $6(9.5)$ & $54(85.7)$ & $\begin{array}{l}3 \\
(4.8)\end{array}$ & \\
\hline \multirow{4}{*}{$\begin{array}{l}\text { Radiation dose } \\
\text { received from } \\
\text { occupational } \\
\text { radiation exposure } \\
\text { Learning new Protocols } \\
\text { \& Procedures }\end{array}$} & Present & $35(20.3)$ & 132(76.7) & $\begin{array}{l}5 \\
(2.9)\end{array}$ & 0.189 \\
\hline & Absent & $5(9.4)$ & $46(86.8)$ & $\begin{array}{l}2 \\
(3.8)\end{array}$ & \\
\hline & Present & $24(25.8)$ & $68(73.1)$ & $\begin{array}{l}1 \\
(1.1)\end{array}$ & $0.014^{\mathrm{a}}$ \\
\hline & Absent & $16(12.1)$ & $110(83.3)$ & $\begin{array}{l}6 \\
(4.5)\end{array}$ & \\
\hline \multirow[t]{2}{*}{$\begin{array}{l}\text { Lack of supporting staff } \\
\text { cooperation }\end{array}$} & Present & $38(20.4)$ & $143(76.9)$ & $\begin{array}{l}5 \\
(2.7)\end{array}$ & 0.063 \\
\hline & Absent & $2(5.1)$ & 35(89.7) & $\begin{array}{l}2 \\
(5.1)\end{array}$ & \\
\hline \multirow{2}{*}{$\begin{array}{l}\text { Lack of cooperation } \\
\text { with Other Nuclear } \\
\text { Medicine Technical } \\
\text { Professionals }\end{array}$} & Present & $34(20.9)$ & $125(76.7)$ & $\begin{array}{l}4 \\
(2.5)\end{array}$ & 0.110 \\
\hline & Absent & $6(9.7)$ & $53(85.5)$ & $\begin{array}{l}3 \\
(4.8)\end{array}$ & \\
\hline \multirow{2}{*}{$\begin{array}{l}\text { Lack of cooperation } \\
\text { with Nuclear } \\
\text { Medicine Physician }\end{array}$} & Present & $29(16.7)$ & $140(80.5)$ & $\begin{array}{l}5 \\
(2.9)\end{array}$ & 0.654 \\
\hline & Absent & $11(21.6)$ & $38(74.5)$ & $\begin{array}{l}2 \\
(3.9)\end{array}$ & \\
\hline \multirow{2}{*}{$\begin{array}{l}\text { Lack of Nuclear } \\
\text { medicine Technical } \\
\text { professionals }\end{array}$} & Present & $37(20.4)$ & $138(76.2)$ & $\begin{array}{l}6 \\
(3.3)\end{array}$ & 0.092 \\
\hline & Absent & $3(6.8)$ & $40(90.9)$ & $\begin{array}{l}1 \\
(2.3)\end{array}$ & \\
\hline
\end{tabular}

Table 3 (continued)

\begin{tabular}{|c|c|c|c|c|c|}
\hline \multirow[t]{2}{*}{ Domain } & \multirow[t]{2}{*}{ Stress } & \multicolumn{3}{|c|}{ Qualification } & \multirow{2}{*}{$\begin{array}{l}\mathrm{P} \\
\text { value }\end{array}$} \\
\hline & & Graduate & Postgraduate & $\mathrm{PhD}$ & \\
\hline \multirow[t]{2}{*}{$\begin{array}{l}\text { Institute Management } \\
\text { Pressure }\end{array}$} & Present & $35(18.5)$ & $150(79.4)$ & $\begin{array}{l}4 \\
(2.1)\end{array}$ & 0.127 \\
\hline & Absent & $5(13.9)$ & $28(77.8)$ & $\begin{array}{l}3 \\
(8.3)\end{array}$ & \\
\hline \multirow[t]{2}{*}{$\begin{array}{l}\text { Incomplete Procedure } \\
\text { Requisition Form }\end{array}$} & Present & $36(20.2)$ & $136(76.4)$ & $\begin{array}{l}6 \\
(3.4)\end{array}$ & 0.146 \\
\hline & Absent & $4(8.5)$ & $42(89.4)$ & $\begin{array}{l}1 \\
(2.1)\end{array}$ & \\
\hline \multirow[t]{2}{*}{ Salary issues } & Present & $33(19.8)$ & $130(77.8)$ & $\begin{array}{l}4 \\
(2.4)\end{array}$ & 0.268 \\
\hline & Absent & $7(12.1)$ & $48(82.8)$ & $\begin{array}{l}3 \\
(5.2)\end{array}$ & \\
\hline \multirow[t]{2}{*}{$\begin{array}{l}\text { Uncertainty of Job } \\
\text { security }\end{array}$} & Present & $35(19.9)$ & $137(77.8)$ & $\begin{array}{l}4 \\
(2.3)\end{array}$ & 0.135 \\
\hline & Absent & $5(10.2)$ & 41(83.7) & $\begin{array}{l}3 \\
(6.1)\end{array}$ & \\
\hline
\end{tabular}

${ }^{\mathrm{a}} \mathrm{p}$ value is less than 0.05 .

modality setup. However, procedure requisition form shows statistically did not report statistically significant difference but few domains like lack of radiation safety equipment, handling non-cooperative patients, handling paediatric patients, institute management pressure, incomplete procedure requisition form showed statistically significant difference among different type of workplace $(\mathrm{p}<0.05)$.

\section{Discussion}

To the best of our knowledge, this is the first study in India exploring the level of occupational stress experienced by Nuclear Medicine Technical professionals working in India. Wherein the effect of age, gender, designation, qualification, institution type, year of work experience, workplace set up and number of qualified technical nuclear professional was studied on 23 occupational stressors as described earlier.

In Covid 19 pandemic, healthcare professionals are exposed to various stressor from working environment which lead to further increase of stress to the highest among these professionals. ${ }^{18}$ In this study, males experienced more stress as compared to female but there was no statistical significance in between gender. However, study conducted by Sechrist and Frazer on 59 nuclear medicine technologists in United States reported the significant difference among gender for stress. ${ }^{14}$ This difference could be due to the fact that Sechrist and Frazer conducted the study in United States which is a high-income country whereas the present study was conducted in India which is a low and middle income country.

A multicentric study conducted by Ugwu AC in south east Nigeria among radiographers reported the highest stress level among 20-40 years of age ${ }^{19}$ but there is no significant difference between age groups and the same findings have been emerged in present study as occupational stress was highest among 21-30 years of age group. This higher level of occupational stress in this age group could be due to the lack of capacity building or training and lack of occupational exposure. ${ }^{19}$ Freshers tend to switch job continuously due to which they are still in process of learning the protocols and procedures in a continuously changing work environment and so their stress levels tend to be high.

There is a statistically significant difference for participant's qualification in few domains like preparation of patients, documentation work, learning new procedures and protocols. Stress was more among those holding post graduate degrees, this may be due to the fact that there is no segregation of job responsibilities between a graduate and a postgraduate as postgraduates holds the utmost duties of the working environment. The burden of the work load and the documentation work being up to date falls more on the postgraduates as compared to 
Table 4

Year of experience wise stress among study participants.

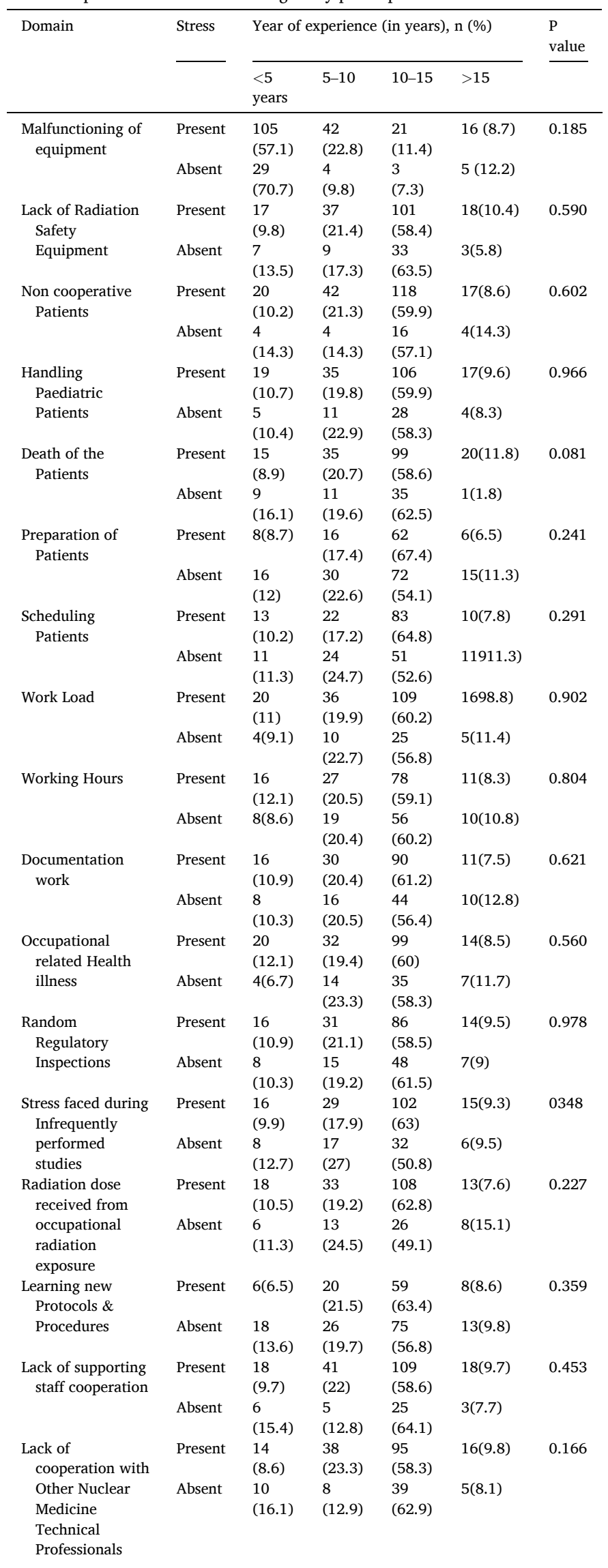

Table 4 (continued)

\begin{tabular}{|c|c|c|c|c|c|c|}
\hline \multirow[t]{2}{*}{ Domain } & \multirow[t]{2}{*}{ Stress } & \multicolumn{4}{|c|}{ Year of experience (in years), n (\%) } & \multirow{2}{*}{$\begin{array}{l}\mathrm{P} \\
\text { value }\end{array}$} \\
\hline & & $\begin{array}{l}<5 \\
\text { years }\end{array}$ & $5-10$ & $10-15$ & $>15$ & \\
\hline \multirow{2}{*}{$\begin{array}{l}\text { Lack of } \\
\text { cooperation with } \\
\text { Nuclear } \\
\text { Medicine } \\
\text { Physician }\end{array}$} & Present & $\begin{array}{l}17 \\
(9.8)\end{array}$ & $\begin{array}{l}39 \\
(22.4)\end{array}$ & $\begin{array}{l}101 \\
(58)\end{array}$ & $17(9.8)$ & \multirow[t]{2}{*}{0.478} \\
\hline & Absent & $\begin{array}{l}7 \\
(13.7)\end{array}$ & $\begin{array}{l}7 \\
(13.7)\end{array}$ & $\begin{array}{l}33 \\
(64.7)\end{array}$ & $4(7.8)$ & \\
\hline \multirow{2}{*}{$\begin{array}{l}\text { Lack of Nuclear } \\
\text { medicine } \\
\text { Technical } \\
\text { professionals }\end{array}$} & Present & $\begin{array}{l}18 \\
(9.9)\end{array}$ & $\begin{array}{l}39 \\
(21.5)\end{array}$ & $\begin{array}{l}106 \\
(58.6)\end{array}$ & 18(9.9) & \multirow[t]{2}{*}{0.676} \\
\hline & Absent & $\begin{array}{l}6 \\
(13.6)\end{array}$ & $\begin{array}{l}7 \\
(15.9)\end{array}$ & $\begin{array}{l}28 \\
(63.6)\end{array}$ & $3(6.8)$ & \\
\hline \multirow{2}{*}{$\begin{array}{l}\text { Institute } \\
\quad \text { Management } \\
\text { Pressure }\end{array}$} & Present & $\begin{array}{l}21 \\
(11.1)\end{array}$ & $\begin{array}{l}39 \\
(20.6)\end{array}$ & $\begin{array}{l}111 \\
(58.7)\end{array}$ & $18(9.5)$ & \multirow[t]{2}{*}{0.936} \\
\hline & Absent & $3(8.3)$ & $\begin{array}{l}7 \\
(19.4)\end{array}$ & $\begin{array}{l}23 \\
(63.9)\end{array}$ & $3(8.3)$ & \\
\hline \multirow{2}{*}{$\begin{array}{l}\text { Incomplete } \\
\text { Procedure } \\
\text { Requisition Form }\end{array}$} & Present & $16(9)$ & $\begin{array}{l}40 \\
(22.5)\end{array}$ & $\begin{array}{l}105 \\
(59)\end{array}$ & $17(9.6)$ & \multirow[t]{2}{*}{0.256} \\
\hline & Absent & $8(17)$ & $\begin{array}{l}6 \\
(12.8)\end{array}$ & $\begin{array}{l}29 \\
(61.7)\end{array}$ & $4(8.5)$ & \\
\hline \multirow[t]{2}{*}{ Salary issues } & Present & $\begin{array}{l}21 \\
(12.6)\end{array}$ & $\begin{array}{l}35 \\
(21)\end{array}$ & $\begin{array}{l}98 \\
(58.7)\end{array}$ & $13(7.8)$ & \multirow[t]{2}{*}{0.257} \\
\hline & Absent & $3(5.2)$ & $\begin{array}{l}11 \\
(19)\end{array}$ & $\begin{array}{l}36 \\
(62.1)\end{array}$ & $8(13.8)$ & \\
\hline \multirow[t]{2}{*}{$\begin{array}{l}\text { Uncertainty of Job } \\
\text { security }\end{array}$} & Present & $\begin{array}{l}19 \\
(10.8)\end{array}$ & $\begin{array}{l}35 \\
(19.9)\end{array}$ & $\begin{array}{l}109 \\
(61.9)\end{array}$ & $13(7.4)$ & \multirow[t]{2}{*}{0.242} \\
\hline & Absent & $\begin{array}{l}5 \\
(10.2)\end{array}$ & $\begin{array}{l}11 \\
(22.4)\end{array}$ & $\begin{array}{l}25 \\
(51)\end{array}$ & $8(16.3)$ & \\
\hline
\end{tabular}

*p value is less than 0.05 .

graduates. Among the PhD degree holders, stress was observed for certain occupational stressors but less in comparison to the graduate and post graduates, the reasons for the difference could be due to difference in sample size.

In our study, stress was seen to be higher in participants having 10-15 years of experience as compared to other groups, this could be attributed to few external factors like work-life balance (conflicting demands of work and home), ${ }^{2}$ COVID-19 situation which could reflect at work or could be due to long-term exposure to the patient suffering at the workplace, resulting in emotional exhaustion. No statistical significance was found for years of work experience among the professionals which refutes the findings of the study conducted by Sechrist and Frazer in USA which showed that there was a greater statistical significance between years of work experience for stressors such as scheduling of patients, equipment malfunction and add-on exams. ${ }^{14}$

Higher percentage of professionals working in multi-modality setup reported more stress as compared to those working in a single modality setup. Stress was observed for handling non corporative patients, paediatric patients, and lack of radiation safety equipment, institute management pressure and incomplete procedure requisition form. This could be attributed to some centre or hospital hiring only a single qualified technical professional to handle the entire department which consists of many modalities so handling each and every modality while checking its daily equipment quality test, performing the patient's scans along with other responsibilities could be stressful. There is no literature available on level of stress based on the single or multi-modality setup in India.

Previous studies on occupational stressors experienced by Nuclear medicine allied health discipline in the United States suggested equipment malfunctions, add-on examinations, uncooperative physicians, lack of staff, and uncooperative patients were the top 5 stressors ${ }^{13,14}$ whereas in our study the top 5 stressors were death of patients, lack of supporting staff cooperation, institute management pressure, lack of radiation safety equipment, uncertainty of job security. While death of a patient injected with radioactivity is rare but appropriate education and training needs to be imparted at the education training level. Most setup in India are now trying to be up to date with radiation safety 
Table 5

Workplace wise stress among study participants.

\begin{tabular}{|c|c|c|c|c|}
\hline \multirow[t]{2}{*}{ Domain } & \multirow[t]{2}{*}{ Stress } & \multicolumn{2}{|c|}{ Type of workplace } & \multirow{2}{*}{$\begin{array}{l}\mathrm{P} \\
\text { value }\end{array}$} \\
\hline & & $\begin{array}{l}\text { Multi- } \\
\text { modality } \\
\text { setup }\end{array}$ & $\begin{array}{l}\text { Single } \\
\text { modality } \\
\text { setup }\end{array}$ & \\
\hline \multirow[t]{2}{*}{ Malfunctioning of equipment } & Present & $133(72.3)$ & $51(27.7)$ & \multirow[t]{2}{*}{0.665} \\
\hline & Absent & $31(75.6)$ & $10(24.4)$ & \\
\hline \multirow{2}{*}{$\begin{array}{l}\text { Lack of Radiation Safety } \\
\text { Equipment }\end{array}$} & Present & $132(76.3)$ & $41(23.7)$ & \multirow[t]{2}{*}{$0.036^{\mathrm{a}}$} \\
\hline & Absent & $32(61.5)$ & $20(38.5)$ & \\
\hline \multirow[t]{2}{*}{ Non cooperative Patients } & Present & $149(75.6)$ & $48(24.4)$ & \multirow[t]{2}{*}{$0.014^{\mathrm{a}}$} \\
\hline & Absent & $15(53.6)$ & $13(46.4)$ & \\
\hline \multirow[t]{2}{*}{ Handling Paediatric Patients } & Present & $137(77.4)$ & $40(22.6)$ & \multirow[t]{2}{*}{$0.003^{\mathrm{a}}$} \\
\hline & Absent & $27(56.2)$ & $21(43.8)$ & \\
\hline \multirow[t]{2}{*}{ Death of the Patients } & Present & $128(75.7)$ & $41(24.3)$ & \multirow[t]{2}{*}{0.095} \\
\hline & Absent & $36(64.3)$ & $20(35.7)$ & \\
\hline \multirow[t]{2}{*}{ Preparation of Patients } & Present & $71(77.2)$ & $21(22.8)$ & \multirow[t]{2}{*}{0.229} \\
\hline & Absent & 93(69.9) & $40(30.1)$ & \\
\hline \multirow[t]{2}{*}{ Scheduling Patients } & Present & 92(71.9) & $36(28.1)$ & \multirow[t]{2}{*}{0.694} \\
\hline & Absent & $72(74.2)$ & $25(25.8)$ & \\
\hline \multirow[t]{2}{*}{ Work Load } & Present & $135(74.6)$ & $46(25.4)$ & \multirow[t]{2}{*}{0.246} \\
\hline & Absent & $29(65.9)$ & $15(34.1)$ & \\
\hline \multirow[t]{2}{*}{ Working Hours } & Present & $97(73.5)$ & $35(26.5)$ & \multirow[t]{2}{*}{0.811} \\
\hline & Absent & $67(72)$ & $26(28)$ & \\
\hline Documentation work & Present & $108(73.5)$ & $39(26.5)$ & 0.788 \\
\hline & Absent & $56(71.8)$ & $22(28.2)$ & \\
\hline Occupational related Health & Present & $125(75.8)$ & $40(24.2)$ & 0.108 \\
\hline illness & Absent & $39(35)$ & $21(35)$ & \\
\hline Random Regulatory & Present & $102(69.4)$ & $45(30.6)$ & 0.105 \\
\hline Inspections & Absent & $62(79.5)$ & $16(20.5)$ & \\
\hline Stress faced during & Present & $121(74.7)$ & $41(25.3)$ & 0.329 \\
\hline $\begin{array}{l}\text { Infrequently performed } \\
\text { studies }\end{array}$ & Absent & $43(68.3)$ & $20(31.7)$ & \\
\hline Radiation dose received from & Present & $128(74.4)$ & $44(28.6)$ & 0.352 \\
\hline $\begin{array}{l}\text { occupational radiation } \\
\text { exposure }\end{array}$ & Absent & $36(67.9)$ & $17(32.1)$ & \\
\hline Learning new Protocols \& & Present & $67(72)$ & $26(28)$ & 0.811 \\
\hline Procedures & Absent & $97(73.5)$ & $35(26.5)$ & \\
\hline Lack of supporting staff & Present & $139(74.7)$ & $47(25.3)$ & 0.175 \\
\hline cooperation & Absent & $25(64.1)$ & $14(35.9)$ & \\
\hline Lack of cooperation with & Present & $124(76.1)$ & $29(23.9)$ & 0.081 \\
\hline Other Nuclear Medicine & Absent & $40(64.5)$ & $22(35.5)$ & \\
\hline Technical Professionals & & & & \\
\hline Lack of cooperation with & Present & $131(75.3)$ & $43(24.7)$ & 0.135 \\
\hline Nuclear Medicine Physician & Absent & $33(64.7)$ & $18(35.3)$ & \\
\hline Lack of Nuclear medicine & Present & $136(75.1)$ & $45(24.9)$ & 0.124 \\
\hline Technical professionals & Absent & $28(63.6)$ & $16(36.4)$ & \\
\hline Institute Management & Present & $144(76.2)$ & $45(23.8)$ & $0.011^{\mathrm{a}}$ \\
\hline Pressure & Absent & $20(55.6)$ & $16(44.4)$ & \\
\hline Incomplete Procedure & Present & $138(77.5)$ & $40(22.5)$ & $0.002^{\mathrm{a}}$ \\
\hline Requisition Form & Absent & $26(55.3)$ & $21(44.7)$ & \\
\hline Salary issues & Present & $120(71.9)$ & $47(28.1)$ & 0.554 \\
\hline & Absent & $44(75.9)$ & $14(24.1)$ & \\
\hline Uncertainty of Job security & Present & $130(73.9)$ & $46(26.1)$ & 0.533 \\
\hline & Absent & $34(69.4)$ & $15(30.6)$ & \\
\hline
\end{tabular}

${ }^{\mathrm{a}} \mathrm{p}$ value is less than 0.05 .

equipment's there are some setup still which needs proper radiation safety equipment's which is a strong necessity as professionals work directly with open radiation sources in Nuclear medicine. As this survey was conducted during the COVID-19 pandemic, the job security was uncertain which could a factor be for it to be in the top 5 stressors.

\subsection{Strength}

As this is the first study conducted among nuclear medicine professionals in India, this study would help in estimation of stress among nuclear medicine professionals at workplace. This study also identifies the minor domains in the workplace which act as a stressors at their workplace. This survey was conducted using a validated, pre-tested tool, self-rated questionnaire which limits the observation bias. To maintain the confidentiality and anonymity of the study participants, no personal information or identifiers were collected.

\section{Conclusion}

The study was aimed at assessing the prevalence of occupational stress and to identify the specific occupational stressors among nuclear medicine technical professionals across the country. The prevalence of occupational stress was $89.3 \%$ among study participants which is on higher side. Work place setup, age and qualification were the factors that had a significant influence on the stress experienced. Overall, the levels of occupational stress reported was high, emphasis on adopting strategies to create a less stressful work environment, increase job satisfaction and reduce stress by providing stress relievers and incentives needs to be taken by the employers.

\section{Funding source}

The authors didn't receive any funding for this work.

\section{Acknowledgement}

The authors want to acknowledge Dr. Shivanand Bhushan, Associate Professor, MAHE, Mr. Amit Nautiyal, Scientific Officer, TATA Memorial Hospital, Mumbai, Dr. Rohit Ravi, Assistant Professor, MAHE for their thorough support in validating the questionnaire and reviewing the final manuscript.

\section{References}

1 Beehr TA, Newman JE. Job stress, employee health, and organizational effectiveness: a facet analysis, model, and literature review1. Person Psychol. 1978 Dec 1;31(4): 665-699. https://doi.org/10.1111/j.1744-6570.1978.tb02118.x.

2 World Health Organization. Occupational health: stress at the workplace. https ://www.who.int/news-room/q-a-detail/ccupational-health-stress-at-the-workplace; 2020. Accessed March 25, 2021.

3 Rajgopal T. Mental well-being at the workplace. Indian J Occup Environ Med. 2010 Sep;14(3):63-65. https://pubmed.ncbi.nlm.nih.gov/21461156.

4 Sathiya N, Ruwaidha R, Nusrath FS, Fathima F, Gomathy T, Shailendra HK. Perceived stress levels and its sources among doctors and nurses working in a tertiary care teaching hospital. Kancheepuram, Tamil Nadu. Ntl J Community Med. 2016;7(7): 603-608.

5 McNamee R, Keen RI, Corkill CM. Morbidity and early retirement among anaesthetists and other specialists. Anaesthesia. 1987:42(2):133-140.

6 Gestal JJ. Occupational hazards in hospitals: accidents, radiation, exposure to noxious chemicals, drug addiction and psychic problems, and assault. Occup Environ Med. 1987;44(8):510-520.

7 Burke RJ, Richardsen AM. Sources of satisfaction and stress among Canadian physicians. Psychol Rep. 1990;67(3):1335-1344.

8 Cooper CL, Rout U, Faragher B. Mental health, job satisfaction, and job stress among general practitioners. Br Med J. 1989;298(6670):366-370.

9 Wong J. Doctors and stress. Med Bull. 2008;13(6):4-7.

10 Vizheh M, Qorbani M, Arzaghi SM, Muhidin S, Javanmard Z, Esmaeili M. The mental health of healthcare workers in the COVID-19 pandemic: a systematic review. J Diabetes Metab Disord. 2020;19(2):1-12.

11 Cabarkapa S, Nadjidai SE, Murgier J, Ng CH. The psychological impact of COVID-19 and other viral epidemics on frontline healthcare workers and ways to address it: a rapid systematic review. Brain, Behav immunity-health. 2020, 100144.

12 SNMMI-TS Scope of Practice Task Forc. Nuclear medicine technologist scope of practice and performance standards. J Nucl Med Technol. 2017;45:325-336.

13 Frazer GH, Sechrist SR. A comparison of occupational stressors in selected allied health disciplines. Health Care Superv. 1994;13(1):53-65.

14 Sechrist SR, Frazer GH. Identification and ranking of stressors in nuclear medicine technology. J Nucl Med Technol. 1990;18(1):44-48.

15 Cai H, Tu B, Ma J, et al. Psychological impact and coping strategies of frontline medical staff in hunan between january and march 2020 during the outbreak of coronavirus disease 2019 (COVID-19) in hubei, China. Med Sci Mon Int Med J Exp Clin Res. 2020 Apr 15;26, e924171-e924171. https://pubmed.ncbi.nlm.nih.gov /32291383.

16 World Medical Association. World Medical Association Declaration of Helsinki: ethical principles for medical research involving human subjects. J Am Med Assoc. 2013 Nov;310(20):2191-2194.

17 Polit DF, Beck CT. The content validity index: are you sure you know what's being reported? Critique and recommendations. Res Nurs Health. 2006 Oct;29(5):489-497.

18 Mathur S, Sharma D, Solanki RK, Goyal MK. Stress related disorders in healthcare workers in COVID-19 pandemic-A cross sectional study from India. Indian $J$ Med Specialities. 2020;11(4):181.

19 Ugwu AC, Erondu OF, Umeano U. Psychosocial stress and its predictors among radiographers in south-eastern Nigeria. SOUTH AFRICAN Radiogr. 2011;49(2).

20 Ravi R, Gunjawate D, Ayas M. Audiology occupational stress experienced by audiologists practicing in India. International Journal of Audiology. 2015;54:131-135. 